\title{
Revising Distributed UNITY Programs is NP-Complete ${ }^{\star}$
}

\author{
Borzoo Bonakdarpour and Sandeep S. Kulkarni \\ Department of Computer Science and Engineering \\ Michigan State University \\ East Lansing, MI 48824, U.S.A. \\ \{borzoo, sandeep\}@cse.msu.edu
}

\begin{abstract}
We focus on automated revision techniques for adding UNITY properties to distributed programs. We show that unlike centralized programs, where multiple safety properties along with one progress property can be simultaneously added in polynomial-time, addition of only one safety or one progress property to distributed programs is NP-complete. We also propose an efficient symbolic heuristic for adding a leads-to property to a distributed program. We demonstrate the application of this heuristic in automated synthesis of recovery paths in fault-tolerant distributed programs.
\end{abstract}

Keywords: UNITY, Distributed programs, Automated revision, Transformation, Repair, Complexity, Formal methods.

\section{Introduction}

Program correctness is an important aspect and application of formal methods. There are two ways to achieve correctness when designing programs: correctby-verification and correct-by-construction. Applying the former often involves a cycle of design, verification, and subsequently manual repair if the verification step does not succeed. The latter, however, achieves correctness in an automated fashion.

Taking the paradigm of correct-by-construction to extreme leads us to synthesizing programs from their specification. While synthesis from specification is undoubtedly useful, it suffers from lack of reuse. In program revision, on the other hand, one can transform an input program into an output program that meets additional properties. As a matter of fact, such properties are frequently identified during a system's life cycle in practice due to reasons such as incomplete specification, renovation of specification, and change of environment. As a concrete example, consider the case where a program is diagnosed with a failed property by a model checker. In such a case, access to automated transformation

\footnotetext{
* This work was partially sponsored by NSF CAREER CCR-0092724 and ONR Grant N00014-01-1-0744.
} 
methods that revise the program at hand with respect to the failed property is highly advantageous. For such revision to be useful, in addition to satisfaction of new properties, the output program must inevitably preserve existing properties of the input program as well.

In our previous work in this context [8], we focused on revising centralized programs, where processes can read and write all program variables in one atomic step, with respect to UNITY [7] properties. Our interest in UNITY properties is due to the fact that they have been found highly expressive in specifying a large class of programs. In [8], we showed that adding a conjunction of multiple UNITY safety properties (i.e., unless, stable, and invariant) along with one progress property (i.e., leads-to and ensures) can be achieved in polynomial-time. We also showed that the problem becomes NP-complete if we consider simultaneous addition of two progress properties. We emphasize that our revision method in [8] ensures satisfaction of all existing UNITY properties of the input program as well.

In this paper, we shift our focus to distributed programs where processes can read and write only a subset of program variables. We expect the concept of program revision to play a more crucial role in the context of distributed programs, since non-determinism and race conditions make it significantly difficult to assert program correctness. We find somewhat unexpected results about the complexity of adding UNITY properties to distributed programs. In particular, we find that the problem of adding only one UNITY safety property or one progress property to a distributed program is NP-complete in the size of the input program's state space.

The knowledge of these complexity bounds is especially important in building tools for incremental synthesis. In particular, the NP-completeness results demonstrate that tools for revising distributed programs must utilize efficient heuristics to expedite the revision algorithm at the cost of completeness. Moreover, NP-completeness proofs often identify where the exponential complexity lies in the problem. Thus, thorough analysis of proofs is also crucial in devising efficient heuristics.

With this motivation, in this paper, we also propose an efficient symbolic heuristic that adds a leads-to property to a distributed program. We integrate this heuristic with our tool SYCRAFT [5] that is designed for adding faulttolerance to existing distributed programs. Meeting leads-to properties are of special interest in fault-tolerant computing where recovery within a finite number of steps is essential. To this end, one can first augment the program with all possible recovery transitions that it can use. This augmented program clearly does not guarantee that it would recover to a set of legitimate states, although there is a potential to reach the legitimate states from states reached in the presence of faults. In particular, it may continue to execute on a cycle that is entirely outside the legitimate states. Thus, we apply our heuristic for adding a leads-to property to modify the augmented program so that from any state reachable in the presence of faults, the program is guaranteed recovery to its legitimate states within a finite number of steps. A by-product of the heuristic for adding 
leads-to properties is a cycle resolution algorithm. Our experimental results show that this algorithm can also be integrated with state-of-the-art model checkers for assisting in developing programs that are correct-by-construction.

Organization. The rest of the paper is organized as follows. In Section 2, we present the preliminary concepts. Then, we formally state the revision problem in Section 3. Section 4 is dedicated to complexity analysis of addition of UNITY safety properties to distributed programs. In Section 5, we present our results on the complexity of addition of UNITY progress properties. We also present our symbolic heuristic and experimental results in Section 5. Related work is discussed in Section 6. Finally, we conclude in Section 7.

\section{Preliminary Concepts}

In this section, we formally define the notion of distributed programs. We also reiterate the concept of UNITY properties introduced by Chandy and Misra [7].

\subsection{Distributed Programs}

Intuitively, we define a distributed program in terms of a set of processes. Each process is in turn specified by a state-transition system and is constrained by some read/write restrictions over its set of variables.

Let $V=\left\{v_{0}, v_{1} \cdots v_{n}\right\}$ be a finite set of variables with finite domains $D_{0}, D_{1} \cdots D_{n}$, respectively. A state, say $s$, is determined by mapping each variable $v_{i}$ in $V, 0 \leq i \leq n$, to a value in $D_{i}$. We denote the value of a variable $v$ in state $s$ by $v(s)$. The set of all possible states obtained by variables in $V$ is called the state space and is denoted by $\mathcal{S}$. A transition is a pair of states of the form $\left(s_{0}, s_{1}\right)$ where $s_{0}, s_{1} \in \mathcal{S}$.

Definition 1 (state predicate) Let $\mathcal{S}$ be the state space obtained from variables in $V$. A state predicate is a subset of $\mathcal{S}$.

Definition 2 (transition predicate) Let $\mathcal{S}$ be the state space obtained from variables in $V$. A transition predicate is a subset of $\mathcal{S} \times \mathcal{S}$.

Definition 3 (process) A process $p$ is specified by the tuple $\left\langle V_{p}, T_{p}, R_{p}, W_{p}\right\rangle$ where $V_{p}$ is a set of variables, $T_{p}$ is a transition predicate in the state space of $p$ (denoted $\mathcal{S}_{p}$ ), $R_{p}$ is a set of variables that $p$ can read, and $W_{p}$ is a set of variables that $p$ can write such that $W_{p} \subseteq R_{p} \subseteq V_{p}$ (i.e., we assume that $p$ cannot blindly write a variable).

Write restrictions. Let $p=\left\langle V_{p}, T_{p}, R_{p}, W_{p}\right\rangle$ be a process. Clearly, $T_{p}$ must be disjoint from the following transition predicate due to inability of $p$ to change the value of variables that $p$ cannot write:

$$
N W_{p}=\left\{\left(s_{0}, s_{1}\right) \mid v\left(s_{0}\right) \neq v\left(s_{1}\right) \text { where } v \notin W_{p}\right\} .
$$


Read restrictions. Let $p=\left\langle V_{p}, T_{p}, R_{p}, W_{p}\right\rangle$ be a process, $v$ be a variable in $V_{p}$, and $\left(s_{0}, s_{1}\right) \in T_{p}$ where $s_{0} \neq s_{1}$. If $v$ is not in $R_{p}$, then $p$ must include a corresponding transition from all states $s_{0}^{\prime}$ where $s_{0}^{\prime}$ and $s_{0}$ differ only in the value of $v$. Let $\left(s_{0}^{\prime}, s_{1}^{\prime}\right)$ be one such transition. Now, it must be the case that $s_{1}$ and $s_{1}^{\prime}$ are identical except for the value of $v$, and, the value of $v$ must be the same in $s_{0}^{\prime}$ and $s_{1}^{\prime}$. For instance, let $V_{p}=\{a, b\}$ and $R_{p}=\{a\}$. Since $p$ cannot read $b$, the transition $([a=0, b=0],[a=1, b=0])$ and the transition ( $[a=0, b=1],[a=1, b=1]$ ) have the same effect as far as $p$ is concerned. Thus, each transition $\left(s_{0}, s_{1}\right)$ in $T_{p}$ is associated with the following group predicate:

$$
\begin{aligned}
& \operatorname{Group}_{p}\left(s_{0}, s_{1}\right)=\left\{\left(s_{0}^{\prime}, s_{1}^{\prime}\right) \mid\right. \\
&\left(\forall v \notin R_{p}:\left(v\left(s_{0}\right)=v\left(s_{1}\right) \wedge v\left(s_{0}^{\prime}\right)=v\left(s_{1}^{\prime}\right)\right)\right) \wedge \\
&\left.\left(\forall v \in R_{p}:\left(v\left(s_{0}\right)=v\left(s_{0}^{\prime}\right) \wedge v\left(s_{1}\right)=v\left(s_{1}^{\prime}\right)\right)\right)\right\} .
\end{aligned}
$$

Definition 4 (distributed program) A distributed program $\Pi$ is specified by the tuple $\left\langle\mathcal{P}_{\Pi}, \mathcal{I}_{\Pi}\right\rangle$ where $\mathcal{P}_{\Pi}$ is a set of processes and $\mathcal{I}_{\Pi}$ is a set of initial states. Without loss of generality, we assume that the state space of all processes in $\mathcal{P}_{\Pi}$ is identical (i.e., $\left.\forall p, q \in \mathcal{P}_{\Pi}::\left(V_{p}=V_{q}\right) \wedge\left(D_{p}=D_{q}\right)\right)$. Thus, the set of variables (denoted $V_{\Pi}$ ) and state space of program $\Pi$ (denoted $\mathcal{S}_{\Pi}$ ) are identical to the set of variables and state space of processes of $\Pi$, respectively. In this sense, the set $\mathcal{I}_{\Pi}$ of initial states of $\Pi$ is a subset of $\mathcal{S}_{\Pi}$.

Notation. $\quad$ Let $\Pi=\left\langle\mathcal{P}_{\Pi}, \mathcal{I}_{\Pi}\right\rangle$ be a distributed program (or simply a program). The set $\mathcal{T}_{\Pi}$ denotes the collection of transition predicates of all processes of $\Pi$, i.e., $\mathcal{T}_{\Pi}=\bigcup_{p \in \mathcal{P}_{\Pi}} T_{p}$.

Definition 5 (computation) Let $\Pi=\left\langle\mathcal{P}_{\Pi}, \mathcal{I}_{\Pi}\right\rangle$ be a program. An infinite sequence of states $\bar{s}=\left\langle s_{0}, s_{1} \cdots\right\rangle$ is a computation of $\Pi$ iff the following three conditions are satisfied: (1) $s_{0} \in \mathcal{I}_{\Pi},(2) \forall i \geq 0:\left(s_{i}, s_{i+1}\right) \in \mathcal{T}_{\Pi}$, and (3) if $\bar{s}$ reaches a terminating state $s_{l}$ where there does not exist $s$ such that $s \neq s_{l}$ and $\left(s_{l}, s\right) \in \mathcal{T}_{\Pi}$, then we extend $\bar{s}$ to an infinite computation by stuttering at $s_{l}$ using transition $\left(s_{l}, s_{l}\right)$.

Notice that we distinguish between a terminating computation and a deadlocked computation. Precisely, if a computation $\bar{s}$ reaches a terminating state $s_{d}$ such that there exists no process $p$ in $\mathcal{P}_{\Pi}$ where $\left(s_{d}, s\right) \in T_{p}$ for some state $s$, then $s_{d}$ is a deadlock state and $\bar{s}$ is a deadlocked computation. For a distributed program $\Pi=\left\langle\mathcal{P}_{\Pi}, \mathcal{I}_{\Pi}\right\rangle$, we say that a sequence of states $\bar{s}=\left\langle s_{0}, s_{1} \cdots s_{n}\right\rangle$ is a computation prefix of $\Pi$ iff $\forall j \mid 0 \leq j<n:\left(s_{j}, s_{j+1}\right) \in \mathcal{T}_{\Pi}$.

\subsection{UNITY Properties}

UNITY properties are categorized by two classes of safety and progress properties defined next [7].

Definition 6 (UNITY safety properties) Let $P$ and $Q$ be arbitrary state predicates. 
- (Unless) An infinite sequence of states $\bar{s}=\left\langle s_{0}, s_{1} \cdots\right\rangle$ satisfies ' $P$ unless $Q$ ' iff $\forall i \geq 0:\left(s_{i} \in(P \cap \neg Q)\right) \Rightarrow\left(s_{i+1} \in(P \cup Q)\right)$. Intuitively, if $P$ holds in a state of $\bar{s}$, then either (1) $Q$ never holds in $\bar{s}$ and $P$ is continuously true, or (2) $Q$ becomes true and $P$ holds at least until $Q$ becomes true.

- (Stable) An infinite sequence of states $\bar{s}=\left\langle s_{0}, s_{1} \cdots\right\rangle$ satisfies 'stable $P$ ' iff $\bar{s}$ satisfies $P$ unless false. Intuitively, $P$ is stable iff once it becomes true, it remains true forever.

- (Invariant) An infinite sequence of states $\bar{s}=\left\langle s_{0}, s_{1} \cdots\right\rangle$ satisfies 'invariant $P^{\prime}$ iff $s_{0} \in P$ and $\bar{s}$ satisfies stable $P$. An invariant property always holds.

Definition 7 (UNITY progress properties) Let $P$ and $Q$ be arbitrary state predicates.

- (Leads-to) An infinite sequence of states $\bar{s}=\left\langle s_{0}, s_{1} \cdots\right\rangle$ satisfies ' $P$ leads-to $Q^{\prime}$ iff $\left(\forall i \geq 0:\left(s_{i} \in P\right) \Rightarrow\left(\exists j \geq i: s_{j} \in Q\right)\right)$. In other words, if $P$ holds in a state $s_{i}, i \geq 0$, of $\bar{s}$, then there exists a state $s_{j}$ in $\bar{s}, i \leq j$, such that $Q$ holds in $s_{j}$.

- (Ensures) An infinite sequence of states $\bar{s}=\left\langle s_{0}, s_{1} \cdots\right\rangle$ satisfies ' $P$ ensures $Q^{\prime}$ 'iff for all $i, i \geq 0$, if $P \cap \neg Q$ is true in state $s_{i}$, then (1) $s_{i+1} \in(P \cup Q)$, and (2) $\exists j \geq i: s_{j} \in Q$. In other words, if $P$ becomes true in $s_{i}$, there exists a state $s_{j}$ where $Q$ eventually becomes true and $P$ remains true everywhere in between $s_{i}$ and $s_{j}$.

In our formal framework, unlike standard UNITY in which interleaved fairness is assumed, we assume that all program computations are unfair. This assumption is necessary when dealing with addition of UNITY progress properties to programs. We also note that the definition of ensures property is slightly different from that in [7]. Precisely, in Chandy and Misra's definition, $P$ ensures $Q$ implies that (1) $P$ leads-to $Q$, (2) $P$ unless $Q$, and (3) there is at least one action that always establishes $Q$ whenever it is executed in a state where $P$ is true and $Q$ is false. Since, we do not model actions explicitly in our work, we have removed the third requirement. Finally, as described in Subsection 2.1, in this paper, our focus is only on programs with finite state space.

We now define what it means for a program to refine a UNITY property. Note that throughout this paper, we assume that a program and its properties have identical state space.

Definition 8 (refines) Let $\Pi=\left\langle\mathcal{P}_{\Pi}, \mathcal{I}_{\Pi}\right\rangle$ be a program and $\mathcal{L}$ be a UNITY property. We say that $\Pi$ refines $\mathcal{L}$ iff all computations of $\Pi$ are infinite and satisfy $\mathcal{L}$.

Definition 9 (specification) A UNITY specification $\Sigma$ is the conjunction $\bigwedge_{i=1}^{n} \mathcal{L}_{i}$ where each $\mathcal{L}_{i}$ is a UNITY safety or progress property.

One can easily extend the notion of refinement to UNITY specifications as follows. Given a program $\Pi$ and a specification $\Sigma=\bigwedge_{i=1}^{n} \mathcal{L}_{i}$, we say that $\Pi$ refines $\Sigma$ iff for all $i, 1 \leq i \leq n, \Pi$ refines $\mathcal{L}_{i}$. 
Concise representation of safety properties. Observe that the UNITY safety properties can be characterized in terms of a set of bad transitions that should never occur in a program computation. For example, stable $P$ requires that a transition, say $\left(s_{0}, s_{1}\right)$, where $s_{0} \in P$ and $s_{1} \notin P$, should never occur in any computation of a program that refines stable $P$. Hence, for simplicity, in this paper, when dealing with safety UNITY properties of a program $\Pi=\left\langle\mathcal{P}_{\Pi}, \mathcal{I}_{\Pi}\right\rangle$, we assume that they are represented by a transition predicate $\mathcal{B} \subseteq \mathcal{S}_{\Pi} \times \mathcal{S}_{\Pi}$ whose transitions should never occur in any computation.

\section{Problem Statement}

Given are a program $\Pi=\left\langle\mathcal{P}_{\Pi}, \mathcal{I}_{\Pi}\right\rangle$ and a (new) UNITY specification $\Sigma_{n}$. Our goal is to devise an automated method which revises $\Pi$ so that the revised program (denoted $\left.\Pi^{\prime}=\left\langle\mathcal{P}_{\Pi^{\prime}}, \mathcal{I}_{\Pi^{\prime}}\right\rangle\right)$ (1) refines $\Sigma_{n}$, and (2) continues refining its existing UNITY specification $\Sigma_{e}$, where $\Sigma_{e}$ is unknown. Thus, during the revision, we only want to reuse the correctness of $\Pi$ with respect to $\Sigma_{e}$ in the sense that the correctness of $\Pi^{\prime}$ with respect to $\Sigma_{e}$ is derived from ' $\Pi$ refines $\Sigma_{e}$ '.

Intuitively, in order to ensure that the revised program $\Pi^{\prime}$ continues refining the existing specification $\Sigma_{e}$, we constrain the revision problem so that the set of computations of $\Pi^{\prime}$ is a subset of the set of computations of $\Pi$. In this sense, since UNITY properties are not existentially quantified (unlike in CTL), we are guaranteed that all computations of $\Pi^{\prime}$ satisfy the UNITY properties that participate in $\Sigma_{e}$.

Now, we formally identify constraints on $\mathcal{S}_{\Pi^{\prime}}, \mathcal{I}_{\Pi^{\prime}}$, and $\mathcal{T}_{\Pi^{\prime}}$. Observe that if $\mathcal{S}_{\Pi^{\prime}}$ contains states that are not in $\mathcal{S}_{\Pi}$, there is no guarantee that the correctness of $\Pi$ with respect to $\Sigma_{e}$ can be reused to ensure that $\Pi^{\prime}$ refines $\Sigma_{e}$. Also, since $\mathcal{S}_{\Pi}$ denotes the set of all states (not just reachable states) of $\Pi$, removing states from $\mathcal{S}_{\Pi}$ is not advantageous. Likewise, $\mathcal{I}_{\Pi^{\prime}}$ should not have any states that were not there in $\mathcal{I}_{\Pi}$. Moreover, since $\mathcal{I}_{\Pi}$ denotes the set of all initial states of $\Pi$, we should preserve them during the revision. Finally, we require that $\mathcal{T}_{\Pi^{\prime}}$ should be a subset of $\mathcal{T}_{\Pi}$. Note that not all transitions of $\mathcal{T}_{\Pi}$ may be preserved in $\mathcal{T}_{\Pi^{\prime}}$. Hence, we must ensure that $\Pi^{\prime}$ does not deadlock. Based on Definitions 8 and 9, if (i) $\mathcal{T}_{\Pi^{\prime}} \subseteq \mathcal{T}_{\Pi}$, (ii) $\Pi^{\prime}$ does not deadlock, and (iii) $\Pi$ refines $\Sigma_{e}$, then $\Pi^{\prime}$ also refines $\Sigma_{e}$. Thus, the revision problem is formally defined as follows:

Problem Statement 1 Given a program $\Pi=\left\langle\mathcal{P}_{\Pi}, \mathcal{I}_{\Pi}\right\rangle$ and a UNITY specification $\Sigma_{n}$, identify $\Pi^{\prime}=\left\langle\mathcal{P}_{\Pi^{\prime}}, \mathcal{I}_{\Pi^{\prime}}\right\rangle$ such that:

(C1) $\mathcal{S}_{\Pi^{\prime}}=\mathcal{S}_{\Pi}$,

(C2) $\mathcal{I}_{\Pi^{\prime}}=\mathcal{I}_{\Pi}$,

(C3) $\mathcal{T}_{\Pi^{\prime}} \subseteq \mathcal{T}_{\Pi}$, and

(C4) $\Pi^{\prime}$ refines $\Sigma_{n}$.

Note that the requirement of deadlock freedom is not explicitly specified in the above problem statement, as it follows from ' $\Pi$ ' refines $\Sigma_{n}$ '. Throughout the paper, we use 'revision of $\Pi$ with respect to a specification $\Sigma_{n}$ (or property $\mathcal{L}$ )' and 'addition of $\Sigma_{n}$ (respectively, $\mathcal{L}$ ) to $\Pi$ ' interchangeably. 


\section{Adding UNITY Safety Properties to Distributed Programs}

As mentioned in Section 2, UNITY safety properties can be characterized by a transition predicate, say $\mathcal{B}$, whose transitions should occur in no computation of a program. In a centralized setting where processes have no restrictions on reading and writing variables, a program $\Pi=\left\langle\mathcal{P}_{\Pi}, \mathcal{I}_{\Pi}\right\rangle$ can be easily revised with respect to $\mathcal{B}$ by simply (1) removing the transitions in $\mathcal{B}$ from $\mathcal{T}_{\Pi}$, and (2) making newly created deadlock states unreachable [8].

To the contrary, the above approach is not adequate for a distributed setting, as it is sound (i.e., it constructs a correct program), but not complete (i.e., it may fail to find a solution while there exists one). This is due to the issue of read restrictions in distributed programs, which associates each transition of a process with a group predicate. This notion of grouping makes the revision complex, as a revision algorithm has to examine many combinations to determine which group of transitions must be removed and, hence, what deadlock states need to be handled. Indeed, we show that the issue of read restrictions changes the class of complexity of the revision problem entirely.

Instance. A distributed program $\Pi=\left\langle\mathcal{P}_{\Pi}, \mathcal{I}_{\Pi}\right\rangle$ and a UNITY safety specification $\Sigma_{n}$.

Decision problem. Does there exist a program $\Pi^{\prime}=\left\langle\mathcal{P}_{\Pi^{\prime}}, \mathcal{I}_{\Pi^{\prime}}\right\rangle$ such that $\Pi^{\prime}$ meets the constraints of Problem Statement 1 for the above instance?

We now show that the above decision problem is NP-complete by a reduction from the well-known satisfiability problem. The SAT problem is as follows:

Let $x_{1}, x_{2} \cdots x_{N}$ be propositional variables. Given a Boolean formula $y=y_{N+1} \wedge y_{N+2} \cdots y_{M+N}$, where each clause $y_{j}, N+1 \leq$ $j \leq M+N$, is a disjunction of three or more literals, does there exist an assignment of truth values to $x_{1}, x_{2} \cdots x_{N}$ such that $y$ is satisfiable?

We note that the unconventional subscripting of clauses in the above definition of the SAT problem is deliberately chosen to make our proofs simpler.

Theorem 1. The problem of adding a UNITY safety property to a distributed program is NP-complete.

Proof. Since showing membership to NP is straightforward, we only need to show that the problem is NP-hard. Towards this end, we present a polynomialtime mapping from an instance of the SAT problem to a corresponding instance of our revision problem. We construct the instance $\Pi=\left\langle\mathcal{P}_{\Pi}, \mathcal{I}_{\Pi}\right\rangle$ as follows.

Variables. The set of variables of program $\Pi$ and, hence, its processes is $V=\left\{v_{0}, v_{1}, v_{2}, v_{3}, v_{4}\right\}$. The domain of these variables are respectively as follows: $\{-1,0,1\},\{-1,0,1\},\{0,1\},\{0,1\},\{-N \cdots-2,-1,1,2 \cdots M+N\} \cup\left\{j^{i} \mid(1 \leq\right.$ $i \leq N) \wedge(N+1 \leq j \leq M+N)\}$. We note that $j^{i}$ in the last set is not an exponent, but a denotational symbol.

Reachable states. The set of reachable states in our mapping is as follows: 
- For each propositional variable $x_{i}, 1 \leq i \leq N$, in the instance of the SAT problem, we introduce the following states (see Figure 1): $a_{i}, b_{i}, b_{i}^{\prime}, c_{i}, c_{i}^{\prime}, d_{i}$, and $d_{i}^{\prime}$. We require that states $a_{1}$ and $a_{N+1}$ are identical.

- For each clause $y_{j}, N+1 \leq j \leq M+N$, we introduce state $r_{j}$.

- For each clause $y_{j}, N+1 \leq j \leq M+N$, and variable $x_{i}$ in clause $y_{j}$, $1 \leq i \leq N$, we introduce the following states: $r_{j i}, s_{j i}, s_{j i}^{\prime}, t_{j i}$, and $t_{j i}^{\prime}$.

Value assignments. Assignment of values to each variable at reachable states is shown in Figure 1 (denoted by $<v_{0}, v_{1}, v_{2}, v_{3}, v_{4}>$ ). We emphasize that assignment of values in our mapping is the most crucial factor in forming group predicates. For reader's convenience, Table 1 illustrates the assignment of values to variables more clearly.

\begin{tabular}{|c|c|c|c|c|c|}
\hline State / Variable name & $v_{0}$ & $v_{1}$ & $v_{2}$ & $v_{3}$ & $v_{4}$ \\
\hline \hline$a_{i}$ & -1 & 1 & 0 & 1 & $i$ \\
\hline$b_{i}$ & 0 & 0 & 0 & 0 & $-i$ \\
\hline$b_{i}^{\prime}$ & 0 & 0 & 0 & 0 & $i$ \\
\hline$c_{i}$ & 1 & 0 & 1 & 1 & $-i$ \\
\hline$c_{i}^{\prime}$ & 0 & 1 & 1 & 1 & $i$ \\
\hline$d_{i}$ & 0 & 1 & 1 & 1 & $-i$ \\
\hline$d_{i}^{\prime}$ & 1 & 0 & 1 & 1 & $i$ \\
\hline
\end{tabular}

(a)

\begin{tabular}{|c|c|c|c|c|c|}
\hline State / Variable name & $v_{0}$ & $v_{1}$ & $v_{2}$ & $v_{3}$ & $v_{4}$ \\
\hline \hline$r_{j}$ & 0 & 0 & 1 & 0 & $j$ \\
\hline$r_{j i}$ & 0 & 0 & 0 & 0 & $j^{2}$ \\
\hline$s_{j i}$ & 0 & 1 & 1 & 1 & $j^{i}$ \\
\hline$s_{j i}^{\prime}$ & 1 & 0 & 1 & 1 & $j^{2}$ \\
\hline$t_{j i}$ & 1 & -1 & 0 & 1 & $j^{i}$ \\
\hline$t_{j i}^{\prime}$ & -1 & -1 & 0 & 1 & $j^{2}$ \\
\hline
\end{tabular}

(b)

Table 1. Assignment of values to variables in proof of Theorem 1.

Processes. Program $\Pi$ consists of four processes. Formally, $\mathcal{P}_{\Pi}=\left\{p_{1}, p_{2}, p_{3}, p_{4}\right\}$. Transition predicate and read/write restrictions of processes in $\mathcal{P}_{\Pi}$ are as follows:

- Read/write restrictions. The read/write restrictions of processes $p_{1}, p_{2}$, $p_{3}$, and $p_{4}$ are as follows:

- $R_{p_{1}}=\left\{v_{0}, v_{2}, v_{3}\right\}$ and $W_{p_{1}}=\left\{v_{0}, v_{2}, v_{3}\right\}$.

- $R_{p_{2}}=\left\{v_{1}, v_{2}, v_{3}\right\}$ and $W_{p_{2}}=\left\{v_{1}, v_{2}, v_{3}\right\}$.

- $R_{p_{3}}=\left\{v_{0}, v_{1}, v_{2}, v_{3}, v_{4}\right\}$ and $W_{p_{3}}=\left\{v_{0}, v_{1}, v_{2}, v_{4}\right\}$.

- $R_{p_{4}}=\left\{v_{0}, v_{1}, v_{2}, v_{3}, v_{4}\right\}$ and $W_{p_{4}}=\left\{v_{0}, v_{1}, v_{3}, v_{4}\right\}$.

- Transition predicates. For each propositional variable $x_{i}, 1 \leq i \leq N$, we include the following transitions in processes $p_{1}, p_{2}, p_{3}$, and $p_{4}$ (see Figure 1):

- $T_{p_{1}}=\left\{\left(b_{i}^{\prime}, d_{i}^{\prime}\right),\left(b_{i}, c_{i}\right) \mid 1 \leq i \leq N\right\}$.

- $T_{p_{2}}=\left\{\left(b_{i}^{\prime}, c_{i}^{\prime}\right),\left(b_{i}, d_{i}\right) \mid 1 \leq i \leq N\right\}$.

- $T_{p_{3}}=\left\{\left(c_{i}^{\prime}, a_{i+1}\right),\left(c_{i}, a_{i+1}\right),\left(d_{i}^{\prime}, a_{i+1}\right),\left(d_{i}, a_{i+1}\right) \mid 1 \leq i \leq N\right\}$. 

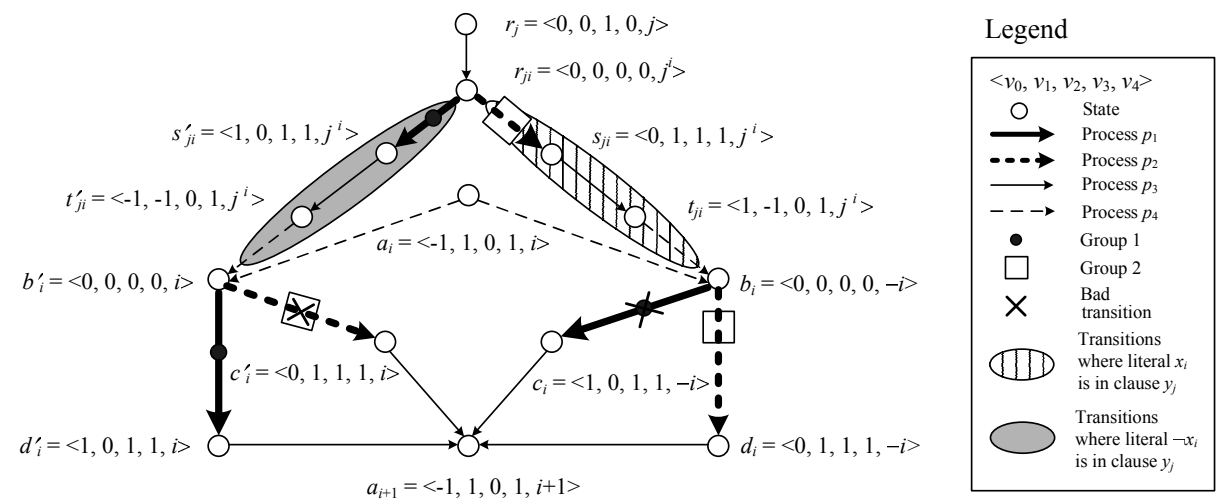

Fig. 1. Mapping SAT to addition of UNITY safety properties.

- $T_{p_{4}}=\left\{\left(a_{i}, b_{i}\right),\left(a_{i}, b_{i}^{\prime}\right) \mid 1 \leq i \leq N\right\}$.

Moreover, corresponding to each clause $y_{j}, N+1 \leq j \leq M+N$, and variable $x_{i}, 1 \leq i \leq N$, in clause $y_{j}$, we include transition $\left(r_{j}, r_{j i}\right)$ in $T_{p_{3}}$ and the following:

- If $x_{i}$ is a literal in clause $y_{j}$, then we include transition $\left(r_{j i}, s_{j i}\right)$ in $T_{p_{2}}$, $\left(s_{j i}, t_{j i}\right)$ in $T_{p_{3}}$, and $\left(t_{j i}, b_{i}\right)$ in $T_{p_{4}}$.

- If $\neg x_{i}$ is a literal in clause $y_{j}$, then we include transition $\left(r_{j i}, s_{j i}^{\prime}\right)$ in $T_{p_{1}}$, $\left(s_{j i}^{\prime}, t_{j i}^{\prime}\right)$ in $T_{p_{3}}$, and $\left(t_{j i}^{\prime}, b_{i}^{\prime}\right)$ in $T_{p_{4}}$.

Note that only for the sake of illustration, Figure 1 shows all possible transitions. However, in order to construct $\Pi$, based on the existence of $x_{i}$ or $\neg x_{i}$ in $y_{j}$, we only include a subset of the transitions.

Initial states. The set $\mathcal{I}_{\Pi}$ of initial states represents clauses of the instance of the SAT problem, i.e., $\mathcal{I}_{\Pi}=\left\{r_{j} \mid N+1 \leq j \leq M+N\right\}$.

Safety property. Let $P$ be a state predicate that contains all reachable states in Figure 1 except $c_{i}$ and $c_{i}^{\prime}$ (i.e., $c_{i}, c_{i}^{\prime} \in \neg P$ ). Thus, the properties stable $P$ and invariant $P$ can be characterized by the transition predicate $\mathcal{B}=$ $\left\{\left(b_{i}, c_{i}\right),\left(b_{i}^{\prime}, c_{i}^{\prime}\right) \mid 1 \leq i \leq N\right\}$. Similarly, let $P$ and $Q$ be two state predicates that contain all reachable states in Figure 1 except $c_{i}$ and $c_{i}^{\prime}$. Thus, the safety property $P$ unless $Q$ can be characterized by $\mathcal{B}$ as well. In our mapping, we let $\mathcal{B}$ represent the safety specification for which $\Pi$ has to be revised.

Before we present our reduction from the SAT problem using the above mapping, we make the following observations regarding the grouping of transitions in different processes:

1. Due to inability of process $p_{1}$ to read variable $v_{4}$, for all $i, 1 \leq i \leq N$, transitions $\left(r_{j i}, s_{j i}^{\prime}\right),\left(b_{i}^{\prime}, d_{i}^{\prime}\right)$, and $\left(b_{i}, c_{i}\right)$ are grouped in $p_{1}$.

2. Due to inability of process $p_{2}$ to read variable $v_{4}$, for all $i, 1 \leq i \leq N$, transitions $\left(r_{j i}, s_{j i}\right),\left(b_{i}, d_{i}\right)$, and $\left(b_{i}^{\prime}, c_{i}^{\prime}\right)$ are grouped in $p_{2}$. 
3. Transitions grouped with the rest of the transitions in Figure 1 are unreachable and, hence, are irrelevant.

Now, we show that the answer to the SAT problem is affirmative if and only if there exists a solution to the revision problem. Thus, we distinguish two cases:

- $(\Rightarrow)$ First, we show that if the given instance of the SAT formula is satisfiable, then there exists a solution that meets the requirements of the revision decision problem. Since the SAT formula is satisfiable, there exists an assignment of truth values to all variables $x_{i}, 1 \leq i \leq N$, such that each $y_{j}$, $N+1 \leq j \leq M+N$, is true. Now, we identify a program $\Pi^{\prime}$, that is obtained by adding the safety property represented by $\mathcal{B}$ to program $\Pi$ as follows.

- The state space of $\Pi^{\prime}$ consists of all the states of $\Pi$, i.e., $\mathcal{S}_{\Pi}=\mathcal{S}_{\Pi^{\prime}}$.

- The initial states of $\Pi^{\prime}$ consists of all the initial states of $\Pi$, i.e., $\mathcal{I}_{\Pi}=\mathcal{I}_{\Pi^{\prime}}$.

- For each variable $x_{i}, 1 \leq i \leq N$, if $x_{i}$ is true, then we include the following transitions: $\left(a_{i}, b_{i}\right)$ in $T_{p_{4}},\left(b_{i}, d_{i}\right)$ in $T_{p_{2}}$, and $\left(d_{i}, a_{i+1}\right)$ in $T_{p_{3}}$.

- For each variable $x_{i}, 1 \leq i \leq N$, if $x_{i}$ is false, then we include the following transitions: $\left(a_{i}, b_{i}^{\prime}\right)$ in $T_{p_{4}},\left(b_{i}^{\prime}, d_{i}^{\prime}\right)$ in $T_{p_{1}}$, and $\left(d_{i}^{\prime}, a_{i+1}\right)$ in $T_{p_{3}}$.

- For each clause $y_{j}, N+1 \leq j \leq M+N$, that contains literal $x_{i}$, if $x_{i}$ is true, we include the following transitions: $\left(r_{j}, r_{j i}\right)$ and $\left(s_{j i}, t_{j i}\right)$ in $T_{p_{3}}$, $\left(r_{j i}, s_{j i}\right)$ in $T_{p_{2}}$, and $\left(t_{j i}, b_{i}\right)$ in $T_{p_{4}}$.

- For each clause $y_{j}, N+1 \leq j \leq M+N$, that contains literal $\neg x_{i}$, if $x_{i}$ is false, we include the following transitions: $\left(r_{j}, r_{j i}\right)$ and $\left(s_{j i}^{\prime}, t_{j i}^{\prime}\right)$ in $T_{p_{3}}$, $\left(r_{j i}, s_{j i}^{\prime}\right)$ in $T_{p_{1}}$, and $\left(t_{j i}^{\prime}, b_{i}^{\prime}\right)$ in $T_{p_{4}}$.

As an illustration, we show the partial structure of $\Pi^{\prime}$, for the formula $\left(x_{1} \vee\right.$ $\left.\neg x_{2} \vee x_{3}\right) \wedge\left(x_{1} \vee x_{2} \vee \neg x_{4}\right)$, where $x_{1}=$ true, $x_{2}=$ false, $x_{3}=$ false, and $x_{4}=$ false, in Figure 2. Notice that states whose all outgoing and incoming transitions are eliminated are not illustrated. Now, we show that $\Pi^{\prime}$ meets the requirements of the Problem Statement 1:

1. The first three constraints of the decision problem are trivially satisfied by construction.

2. We now show that constraint $C 4$ holds. First, it is easy to observe that by construction, there exist no reachable deadlock states in the revised program. Hence, if $\Pi$ refines UNITY specification $\Sigma_{e}$, then $\Pi^{\prime}$ refines $\Sigma_{e}$ as well. Moreover, if a computation of $\Pi^{\prime}$ reaches a state $b_{i}$ for some $i$, from an initial state $r_{j}$ (i.e., $x_{i}$ is true in clause $y_{j}$ ), then that computation cannot violate safety since bad transition $\left(b_{i}, c_{i}\right)$ is removed. This is due to the fact that $\left(b_{i}, c_{i}\right)$ is grouped with transition $\left(r_{j i}, s_{j i}^{\prime}\right)$ and this transition is not included in $\mathcal{T}_{\Pi^{\prime}}$, as literal $x_{i}$ is true in $y_{j}$. Likewise, if a computation of $\Pi^{\prime}$ reaches a state $b_{i}^{\prime}$ for some $i$, from initial state $r_{j}$ (i.e., $x_{i}$ is false in clause $y_{j}$ ), then that computation cannot violate safety since transition $\left(b_{i}^{\prime}, c_{i}^{\prime}\right)$ is removed. This is due to the fact that $\left(b_{i}^{\prime}, c_{i}^{\prime}\right)$ is grouped with transition $\left(r_{j i}, s_{j i}\right)$ and this transition is not included in $\mathcal{T}_{\Pi^{\prime}}$, as $x_{i}$ is false. Thus, $\Pi^{\prime}$ refines $\Sigma_{n}$. 


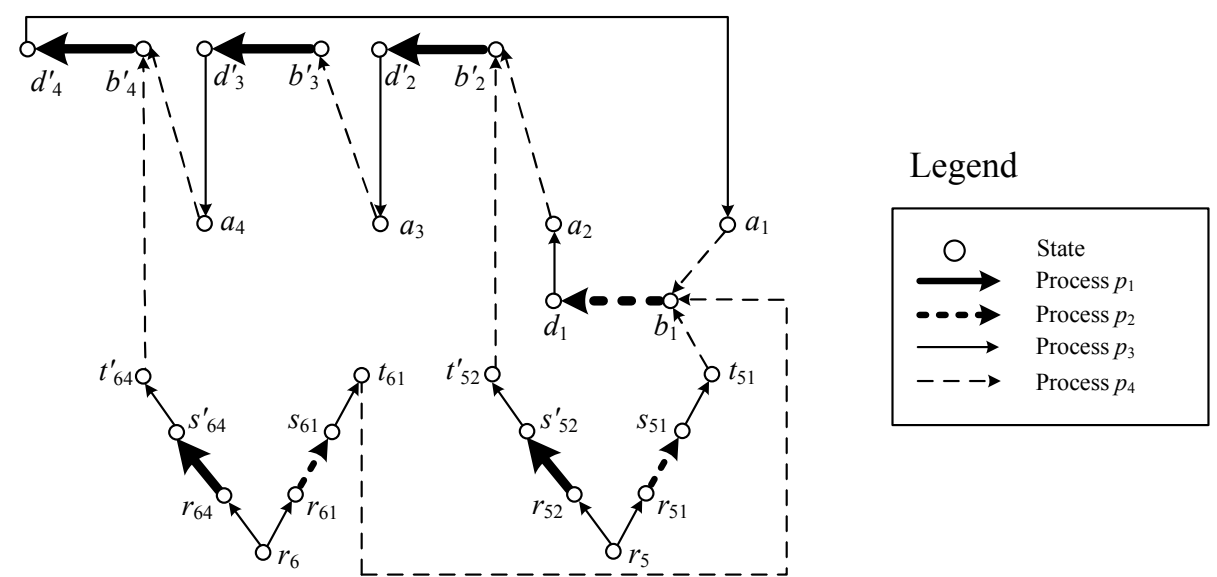

Fig. 2. The structure of the revised program for Boolean formula $\left(x_{1} \vee \neg x_{2} \vee\right.$ $\left.x_{3}\right) \wedge\left(x_{1} \vee x_{2} \vee \neg x_{4}\right)$, where $x_{1}=$ true, $x_{2}=$ false, $x_{3}=$ false, and $x_{4}=$ false.

$-(\Leftarrow)$ Next, we show that if there exists a solution to the revision problem for the instance identified by our mapping from the SAT problem, then the given SAT formula is satisfiable. Let $\Pi^{\prime}$ be the program that is obtained by adding the safety property $\Sigma_{n}$ to program $\Pi$. Now, in order to obtain a solution for SAT, we proceed as follows. If there exists a computation of $\Pi^{\prime}$ where state $b_{i}$ is reachable, then we assign $x_{i}$ the truth value true. Otherwise, we assign the truth value false.

We now show that the above truth assignment satisfies all clauses. Let $y_{j}$ be a clause for some $j, N+1 \leq j \leq M+N$, and let $r_{j}$ be the corresponding initial state in $\mathcal{I}_{\Pi^{\prime}}$. Since $r_{j}$ is an initial state and $\Pi^{\prime}$ cannot deadlock, the transition $\left(r_{j}, r_{j i}\right)$ must be present in $\mathcal{T}_{\Pi^{\prime}}$, for some $i, 1 \leq i \leq N$. By the same argument, there must exist some transition that originates from $r_{j i}$. This transition terminates in either $s_{j i}$ or $s_{j i}^{\prime}$. Observe that $\mathcal{T}_{\Pi^{\prime}}$ cannot have both transitions, as grouping of transitions will include both $\left(b_{i}, c_{i}\right)$ and $\left(b_{i}^{\prime}, c_{i}^{\prime}\right)$ which in turn causes violation of safety by $\Pi^{\prime}$. Now, if the transition from $r_{j i}$ terminates in $s_{j i}$, then clause $y_{j}$ contains literal $x_{i}$ and $x_{i}$ is assigned the truth value true. Hence, $y_{j}$ evaluates to true. Likewise, if the transition from $r_{j i}$ terminates in $s_{j i}^{\prime}$, then clause $y_{j}$ contains literal $\neg x_{i}$ and $x_{i}$ is assigned the truth value false. Hence, $y_{j}$ evaluates to true. Therefore, the assignment of values considered above is a satisfying truth assignment for the given SAT formula.

\section{Adding UNITY Progress Properties to Distributed Programs}

This section is organized as follows. In Subsection 5.1, we show that adding a UNITY progress property to a distributed program is NP-complete. Then, in 
Subsection 5.2, we present a symbolic heuristic for adding a leads-to property to a distributed program.

\subsection{Complexity}

In a centralized setting, where programs have no restriction on reading and writing variables, a program, say $\Pi=\left\langle\mathcal{P}_{\Pi}, \mathcal{I}_{\Pi}\right\rangle$, can be easily revised with respect to a progress property by simply (1) breaking non-progress cycles that prevent a program to eventually reach a desirable state predicate, and (2) removing deadlock states [8]. To the contrary, in a distributed setting, due to the issue of grouping, it matters which transition (and as a result its corresponding group) is removed to break a non-progress cycle.

Instance. A distributed program $\Pi=\left\langle\mathcal{P}_{\Pi}, \mathcal{I}_{\Pi}\right\rangle$ and a UNITY progress property $\Sigma_{n}$.

Decision problem. Does there exist a program $\Pi^{\prime}=\left\langle\mathcal{P}_{\Pi^{\prime}}, \mathcal{I}_{\Pi^{\prime}}\right\rangle$ such that $\Pi^{\prime}$ meets the constraints of Problem Statement 1 for the above instance?

Theorem 2. The problem of adding a UNITY progress property to a distributed program is NP-complete.

Proof. Since showing membership to NP is straightforward, we only show that the problem is NP-hard by a reduction from the SAT problem. First, we present a polynomial-time mapping.

Variables. The set of variables of program $\Pi$ and, hence, its processes is $V=\left\{v_{0}, v_{1}, v_{2}, v_{3}, v_{4}\right\}$. The domain of these variables are respectively as follows: $\{0,1\},\{0,1\},\{-N \cdots-2,-1,1,2 \cdots M+N\} \cup\left\{j^{i} \mid(1 \leq i \leq N) \wedge(N+1 \leq\right.$ $j \leq M+N)\},\{-1,0,1\}$, and $\{-1,0,1\}$.

Reachable states. The set of reachable states in our mapping is as follows:

- For each propositional variable $x_{i}, 1 \leq i \leq N$, we introduce the following states (see Figure 3): $a_{i}, a_{i}^{\prime}, b_{i}, b_{i}^{\prime}, c_{i}, c_{i}^{\prime}, d_{i}, d_{i}^{\prime}, Q_{i}$, and $Q_{i}^{\prime}$.

- For each clause $y_{j}, N+1 \leq j \leq M+N$, we introduce state $r_{j}$.

- For each clause $y_{j}, N+1 \leq j \leq M+N$, and variable $x_{i}, 1 \leq i \leq N$, in clause $y_{j}$, we introduce states $r_{j i}, s_{j i}$, and $s_{j i}^{\prime}$.

Value assignments. Assignment of values to each variable at reachable states is shown in Figure 3 (denoted by $<v_{0}, v_{1}, v_{2}, v_{3}, v_{4}>$ ). For reader's convenience, Table 2 illustrates the assignment of values to variables more clearly.

Processes. Program $\Pi$ consists of four processes. Formally, $\mathcal{P}_{\Pi}=\left\{p_{1}, p_{2}, p_{3}, p_{4}\right\}$. Transition predicate and read/write restrictions of processes in $\mathcal{P}_{\Pi}$ are as follows:

- Read/write restrictions. The read/write restrictions of processes $p_{1}, p_{2}$, $p_{3}$, and $p_{4}$ are as follows:

- $R_{p_{1}}=\left\{v_{0}, v_{1}, v_{3}\right\}$ and $W_{p_{1}}=\left\{v_{0}, v_{1}, v_{3}\right\}$.

- $R_{p_{2}}=\left\{v_{0}, v_{1}, v_{4}\right\}$ and $W_{p_{2}}=\left\{v_{0}, v_{1}, v_{4}\right\}$.

- $R_{p_{3}}=\left\{v_{0}, v_{1}, v_{2}, v_{3}, v_{4}\right\}$ and $W_{p_{3}}=\left\{v_{0}, v_{2}, v_{3}, v_{4}\right\}$.

- $R_{p_{4}}=\left\{v_{0}, v_{1}, v_{2}, v_{3}, v_{4}\right\}$ and $W_{p_{4}}=\left\{v_{1}, v_{2}, v_{3}, v_{4}\right\}$. 


\begin{tabular}{|c|c|c|c|c|c|}
\hline State / Variable name & $v_{0}$ & $v_{1}$ & $v_{2}$ & $v_{3}$ & $v_{4}$ \\
\hline \hline$a_{i}$ & 1 & 0 & $-i$ & -1 & -1 \\
\hline$a_{i}^{\prime}$ & 1 & 0 & $i$ & -1 & 1 \\
\hline$b_{i}$ & 0 & 0 & $-i$ & 0 & 0 \\
\hline$b_{i}^{\prime}$ & 0 & 0 & $i$ & 0 & 0 \\
\hline$c_{i}$ & 1 & 1 & $-i$ & 0 & 1 \\
\hline$c_{i}^{\prime}$ & 1 & 1 & $i$ & 1 & 0 \\
\hline$d_{i}$ & 0 & 1 & $i$ & 1 & -1 \\
\hline$d_{i}^{\prime}$ & 0 & 1 & $-i$ & 1 & 1 \\
\hline$Q_{i}$ & 1 & 1 & $-i$ & 1 & 0 \\
\hline$Q_{i}^{\prime}$ & 1 & 1 & $i$ & 0 & 1 \\
\hline
\end{tabular}

\begin{tabular}{|c|c|c|c|c|c|}
\hline State / Variable name & $v_{0}$ & $v_{1}$ & $v_{2}$ & $v_{3}$ & $v_{4}$ \\
\hline \hline$r_{j}$ & 0 & 1 & $j$ & 1 & 1 \\
\hline$r_{j i}$ & 0 & 0 & $j^{i}$ & 0 & 0 \\
\hline$s_{j i}$ & 1 & 1 & $j^{i}$ & 0 & 1 \\
\hline$s_{j i}^{\prime}$ & 1 & 1 & $j^{i}$ & 1 & 0 \\
\hline
\end{tabular}

(b)

(a)

Table 2. Assignment of values to variables in proof of Theorem 2.

- Transition predicates. For each propositional variable $x_{i}, 1 \leq i \leq N$, we include the following transitions in processes $p_{1}, p_{2}, p_{3}$, and $p_{4}$ (see Figure 3):

- $T_{p_{1}}=\left\{\left(b_{i}^{\prime}, c_{i}^{\prime}\right),\left(b_{i}, Q_{i}\right) \mid 1 \leq i \leq N\right\}$.

- $T_{p_{2}}=\left\{\left(b_{i}, c_{i}\right),\left(b_{i}^{\prime}, Q_{i}^{\prime}\right) \mid 1 \leq i \leq N\right\}$.

- $T_{p_{3}}=\left\{\left(a_{i}, b_{i}\right),\left(a_{i}^{\prime}, b_{i}^{\prime}\right),\left(c_{i}, d_{i}\right),\left(c_{i}^{\prime}, d_{i}^{\prime}\right),\left(Q_{i}, Q_{i}\right),\left(Q_{i}^{\prime}, Q_{i}^{\prime}\right) \mid 1 \leq i \leq N\right\}$.

- $T_{p_{4}}=\left\{\left(d_{i}^{\prime}, b_{i}\right),\left(d_{i}, b_{i}^{\prime}\right) \mid 1 \leq i \leq N\right\}$.

Moreover, corresponding to each clause $y_{j}, N+1 \leq j \leq M+N$, and variable $x_{i}, 1 \leq i \leq N$, in clause $y_{j}$, we include transition $\left(r_{j}, r_{j i}\right)$ in $T_{p_{4}}$ and the following:

- If $x_{i}$ is a literal in clause $y_{j}$, then we include transition $\left(r_{j i}, s_{j i}\right)$ in $T_{p_{2}}$, and $\left(s_{j i}, a_{i}\right)$ in $T_{p_{4}}$.

- If $\neg x_{i}$ is a literal in clause $y_{j}$, then we include transition $\left(r_{j i}, s_{j i}^{\prime}\right)$ in $T_{p_{1}}$ and $\left(s_{j i}^{\prime}, a_{i}^{\prime}\right)$ in $T_{p_{4}}$.

Note that for the sake of illustration, Figure 3 shows all possible transitions. However, in order to construct $\Pi^{\prime}$, based on the existence of $x_{i}$ or $\neg x_{i}$ in $y_{j}$, we only include a subset of transitions.

Initial states. The set $\mathcal{I}_{\Pi}$ of initial states represents clauses of the Boolean formula in the instance of the SAT problem, i.e., $\mathcal{I}_{\Pi}=\left\{r_{j} \mid N+1 \leq j \leq M+N\right\}$. Progress property. In our mapping, the desirable progress property is of the form $\Sigma_{n} \equiv$ (true leads-to $Q$ ), where $Q=\left\{Q_{i}, Q_{i}^{\prime} \mid 1 \leq i \leq N\right\}$ (see Figure 3 ). Observe that $\Sigma_{n}$ is a leads-to as well as an ensures property. This property in Linear Temporal Logic (LTL) is denoted by $\square \diamond Q$ (called always eventually $Q$ ).

Before we present our reduction from the SAT problem using the above mapping, we make the following observations regarding the grouping of transitions in different processes: 


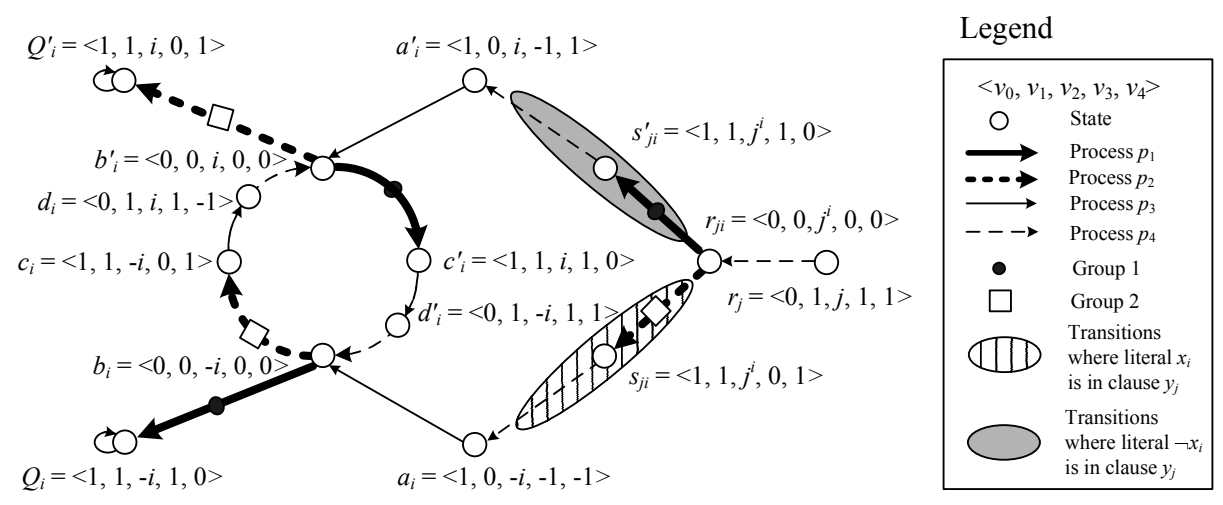

Fig. 3. Mapping SAT to addition of a progress property.

1. Due to inability of process $p_{1}$ to read variable $v_{2}$, for all $i, 1 \leq i \leq N$, transitions $\left(r_{j i}, s_{j i}^{\prime}\right),\left(b_{i}^{\prime}, c_{i}^{\prime}\right)$, and $\left(b_{i}, Q_{i}\right)$ are grouped in process $p_{1}$.

2. Due to inability of process $p_{2}$ to read variable $v_{2}$, for all $i, 1 \leq i \leq N$, transitions $\left(r_{j i}, s_{j i}\right),\left(b_{i}, c_{i}\right)$, and $\left(b_{i}^{\prime}, Q_{i}^{\prime}\right)$ are grouped in process $p_{2}$.

3. Transitions grouped with the rest of the transitions in Figure 3 are unreachable and, hence, are irrelevant.

We distinguish the following two cases for reducing the SAT problem to our revision problem :

- $(\Rightarrow)$ First, we show that if the given instance of the SAT formula is satisfiable, then there exists a solution that meets the requirements of the revision decision problem. Since the SAT formula is satisfiable, there exists an assignment of truth values to all variables $x_{i}, 1 \leq i \leq N$, such that each $y_{j}$, $N+1 \leq j \leq M+N$, is true. Now, we identify a program $\Pi^{\prime}$, that is obtained by adding the progress property $\square \diamond Q$ to program $\Pi$ as follows.

- The state space of $\Pi^{\prime}$ consists of all the states of $\Pi$, i.e., $\mathcal{S}_{\Pi}=\mathcal{S}_{\Pi^{\prime}}$.

- The initial states of $\Pi^{\prime}$ consists of all the initial states of $\Pi$, i.e., $\mathcal{I}_{\Pi}=\mathcal{I}_{\Pi^{\prime}}$.

- For each variable $x_{i}, 1 \leq i \leq N$, if $x_{i}$ is true, then we include the following transitions: $\left(a_{i}, b_{i}\right),\left(c_{i}, d_{i}\right)$, and $\left(Q_{i}^{\prime}, Q_{i}^{\prime}\right)$ in $T_{p_{3}},\left(b_{i}, c_{i}\right)$ and $\left(b_{i}^{\prime}, Q_{i}^{\prime}\right)$ in $T_{p_{2}}$, and, $\left(d_{i}, b_{i}^{\prime}\right)$ in $T_{p_{4}}$.

- For each variable $x_{i}, 1 \leq i \leq N$, if $x_{i}$ is false, then we include the following transitions: $\left(a_{i}^{\prime}, b_{i}^{\prime}\right),\left(c_{i}^{\prime}, d_{i}^{\prime}\right)$, and $\left(Q_{i}, Q_{i}\right)$ in $T_{p_{3}},\left(b_{i}^{\prime}, c_{i}^{\prime}\right)$ and $\left(b_{i}, Q_{i}\right)$ in $T_{p_{1}}$, and, $\left(d_{i}^{\prime}, b_{i}\right)$ in $T_{p_{4}}$.

- For each clause $y_{j}, N+1 \leq j \leq M+N$, that contains literal $x_{i}$, if $x_{i}$ is true, we include transitions $\left(r_{j}, r_{j i}\right)$ and $\left(s_{j i}, a_{i}\right)$ in $T_{p_{4}}$, and, transition $\left(r_{j i}, s_{j i}\right)$ in $T_{p_{2}}$.

- For each clause $y_{j}, N+1 \leq j \leq M+N$, that contains literal $\neg x_{i}$, if $x_{i}$ is false, we include transitions $\left(r_{j}, r_{j i}\right)$ and $\left(s_{j i}^{\prime}, a_{i}^{\prime}\right)$ in $T_{p_{4}}$, and, transition $\left(r_{j i}, s_{j i}^{\prime}\right)$ in $T_{p_{1}}$. 


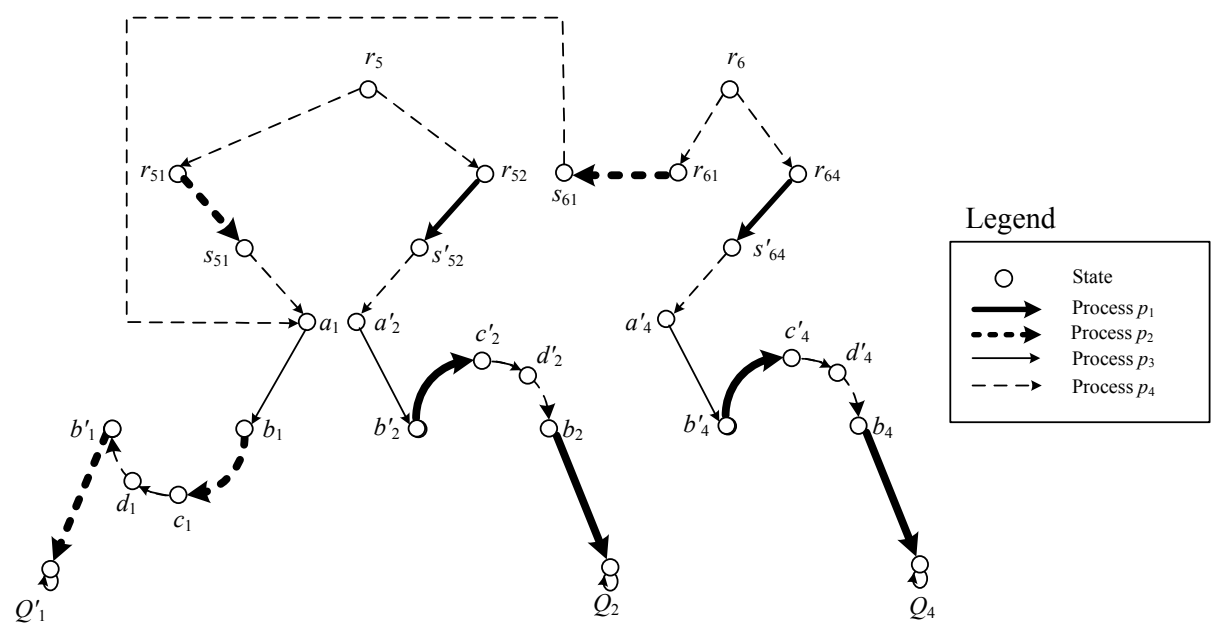

Fig. 4. The structure of the revised program for Boolean formula $\left(x_{1} \vee \neg x_{2} \vee\right.$ $\left.x_{3}\right) \wedge\left(x_{1} \vee x_{2} \vee \neg x_{4}\right)$, where $x_{1}=$ true, $x_{2}=$ false, $x_{3}=$ false, and $x_{4}=$ false.

As an illustration, we show the partial structure of $\Pi^{\prime}$, for the formula $\left(x_{1} \vee\right.$ $\left.\neg x_{2} \vee x_{3}\right) \wedge\left(x_{1} \vee x_{2} \vee \neg x_{4}\right)$, where $x_{1}=$ true, $x_{2}=$ false, $x_{3}=$ false, and $x_{4}=$ false in Figure 4. Notice that states whose all outgoing and incoming transitions are eliminated are not illustrated. Now, we show that $\Pi^{\prime}$ meets the requirements of the Problems Statement 1:

1. The first three constraints of the decision problem are trivially satisfied by construction.

2. We now show that constraint $C 4$ holds. First, it is easy to observe that by construction, there exist no reachable deadlock states in the revised program. Hence, if $\Pi$ refines UNITY specification $\Sigma_{e}$, then $\Pi^{\prime}$ refines $\Sigma_{e}$ as well. Moreover, by construction, all computations of $\Pi^{\prime}$ eventually reach either $Q_{i}$ or $Q_{i}^{\prime}$ and will stutter there. This is due to the fact that if literal $x_{i}$ is true in clause $y_{j}$, then transition $\left(r_{j i}, s_{j i}^{\prime}\right)$ is not included in $\mathcal{T}_{\Pi^{\prime}}$ and, hence, its group-mates $\left(b_{i}^{\prime}, c_{i}^{\prime}\right)$ and $\left(b_{i}, Q_{i}\right)$ are not in $\mathcal{T}_{\Pi^{\prime}}$ as well. Consequently, a computation that starts from $r_{j}$ eventually reaches $Q_{i}^{\prime}$ without meeting a cycle. Likewise, if literal $\neg x_{i}$ is false in clause $y_{j}$, then transition $\left(r_{j i}, s_{j i}\right)$ is not included in $\mathcal{T}_{\Pi^{\prime}}$ and, hence, its group-mates $\left(b_{i}, c_{i}\right)$ and $\left(b_{i}^{\prime}, Q_{i}^{\prime}\right)$ are not in $\mathcal{T}_{\Pi^{\prime}}$ as well. Consequently, a computation that starts from $r_{j}$ eventually reaches $Q_{i}$ without meeting a cycle. Hence, $\Pi^{\prime}$ refines $\Sigma_{n} \equiv \square \diamond Q$.

$-(\Leftarrow)$ Next, we show that if there exists a solution to the revision problem for the instance identified by our mapping from the SAT problem, then the given SAT formula is satisfiable. Let $\Pi^{\prime}$ be the program that is obtained by adding the progress property in $\Sigma_{n} \equiv \square \diamond Q$ to program $\Pi$. Now, in order to obtain a solution for SAT, we proceed as follows. If there exists a computation of $\Pi^{\prime}$ 
where state $a_{i}$ is reachable, then we assign $x_{i}$ the truth value true. Otherwise, we assign the truth value false.

We now show that the above truth assignment satisfies all clauses. Let $y_{j}$ be a clause for some $j, N+1 \leq j \leq M+N$, and let $r_{j}$ be the corresponding initial state in $\mathcal{I}_{\Pi^{\prime}}$. Since $r_{j}$ is an initial state and $\Pi^{\prime}$ cannot deadlock, the transition $\left(r_{j}, r_{j i}\right)$ must be present in $\mathcal{T}_{\Pi^{\prime}}$, for some $i, 1 \leq i \leq N$. By the same argument, there must exist some transition that originates from $r_{j i}$. This transition terminates in either $s_{j i}$ or $s_{j i}^{\prime}$. Observe that $\mathcal{T}_{\Pi^{\prime}}$ cannot have both transitions, as grouping of transitions will include transitions $\left(b_{i}, c_{i}\right)$ and $\left(b_{i}^{\prime}, c_{i}^{\prime}\right)$. If this is the case, $\Pi^{\prime}$ does not refine the property $\square \diamond Q$ due to the existence of cycle $b_{i} \rightarrow c_{i} \rightarrow d_{i} \rightarrow b_{i}^{\prime} \rightarrow c_{i}^{\prime} \rightarrow d_{i}^{\prime} \rightarrow b_{i}$. Thus, there can be one and only one outgoing transition from $r_{j i}$ in $\mathcal{T}_{\Pi^{\prime}}$. Now, if the transition from $r_{j i}$ terminates in $s_{j i}$, then clause $y_{j}$ contains literal $x_{i}$ and $x_{i}$ is assigned the truth value true. Hence, $y_{j}$ evaluates to true. Likewise, if the transition from $r_{j i}$ terminates in $s_{j i}^{\prime}$, then clause $y_{j}$ contains literal $\neg x_{i}$ and $x_{i}$ is assigned the truth value false. Hence, $y_{j}$ evaluates to true. Therefore, the assignment of values considered above is a satisfying truth assignment for the given SAT formula.

\subsection{A Symbolic Heuristic for Adding Leads-To Properties}

We now present a polynomial-time (in the size of the state space) symbolic (BDD ${ }^{1}$-based) heuristic for adding leads-to properties to distributed programs. Leads-to properties have interesting applications in automated addition of recovery for synthesizing fault-tolerant distributed programs.

The NP-hardness reduction presented in the proof of Theorem 2 precisely shows where the complexity of the problem lies in. Indeed, Figure 3 shows that transition $\left(b_{i}, c_{i}\right)$ which can potentially be removed to break the non-progress cycle $b_{i} \rightarrow c_{i} \rightarrow d_{i} \rightarrow b_{i}^{\prime} \rightarrow c_{i}^{\prime} \rightarrow d_{i}^{\prime} \rightarrow b_{i}$ is grouped with the critical transition $\left(r_{j i}, s_{j i}\right)$ which ensures that state $r_{j i}$ and consequently initial state $r_{j}$ are not deadlocked. The same argument holds for transitions $\left(b_{i}^{\prime}, c_{i}^{\prime}\right)$ and $\left(r_{j i}, s_{j i}^{\prime}\right)$. Thus, a heuristic that adds a leads-to property to a distributed program needs to address this issue.

Our heuristic works as follows (cf. Figure 5). The Algorithm Add_LeadsTo takes a distributed program $\Pi=\left\langle\mathcal{P}_{\Pi}, \mathcal{I}_{\Pi}\right\rangle$ and a property $P$ leads-to $Q$ as input, where $P$ and $Q$ are two arbitrary state predicates in the state space of $\Pi$. The algorithm (if successful) returns transition predicate of the derived program $\Pi^{\prime}=$ $\left\langle\mathcal{P}_{\Pi^{\prime}}, \mathcal{I}_{\Pi^{\prime}}\right\rangle$ that refines $P$ leads-to $Q$ as output. In order to transform $\Pi$ to $\Pi^{\prime}$, first, the algorithm ranks states that can be reached from $P$ based on the length of their shortest path to $Q$ (Line 2). Then, it attempts to break non-progress cycles (Lines 3-13). To this end, it first computes the set of cycles that are reachable from $P$ (Line 4). This computation can be accomplished using any

\footnotetext{
${ }^{1}$ Ordered Binary Decision Diagrams [6] represent Boolean formulae as directed acyclic graphs making testing of functional properties such as satisfiability and equivalence straightforward and extremely efficient.
} 


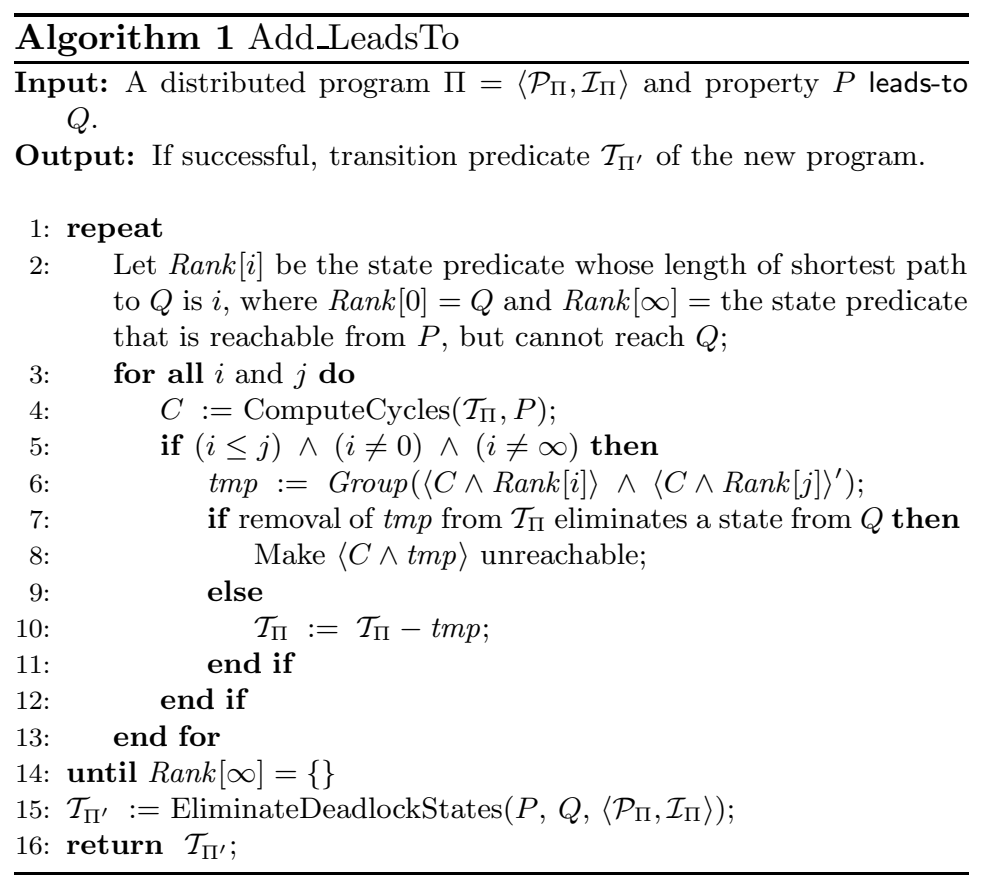

Fig. 5. A symbolic heuristic for adding a leads-to property to a distributed program.

BDD-based cycle detection algorithm. We apply the Emerson-Lie method [10]. Then, the algorithm removes transitions from $\mathcal{T}_{\Pi}$ that participate in a cycle and whose rank of source state is less than or equal to the rank of destination state (Lines 6-10). However, since removal of a transition must take place with its entire group predicate, we do not remove a transition that causes creation of deadlock states in $Q$. Instead, we make the corresponding cycle unreachable (Line 8). This can be done by simply removing transitions that terminate in a state on the cycle. Thus, if removal of a group of transitions does not create new deadlock states in $Q$, the algorithm removes them (Line 10). Finally, since removal of transitions may create deadlock states outside $Q$ but reachable from $P$, we need to eliminate those deadlock states (Line 15). Such elimination can be accomplished using the BDD-based method proposed in [4].

Given $O\left(n^{2}\right)$ complexity of the cycle detection algorithm [10], it is straightforward to observe that the complexity of our heuristic is $O\left(n^{4}\right)$, where $n$ is the size of state space of $\Pi$. In order to evaluate the performance of our heuristic, we have implemented the Algorithm Add_LeadsTo in our tool SYCRAFT [5]. This heuristic can be used for adding recovery in order to synthesize fault-tolerant distributed programs as follows. Let $S$ be a set of legitimate states (e.g., an invariant predicate) and $T$ be the fault-span predicate (i.e., the set of states 


\begin{tabular}{|c|c|c|c|c|c|}
\hline & \multicolumn{2}{|c|}{ Space } & \multicolumn{3}{|c|}{ Time $(s)$} \\
\hline & $\begin{array}{c}\text { reachable } \\
\text { states }\end{array}$ & $\begin{array}{c}\text { memory } \\
\text { (KB) }\end{array}$ & $\begin{array}{c}\text { cycle } \\
\text { detection }\end{array}$ & $\begin{array}{c}\text { pruning } \\
\text { transitions }\end{array}$ & total \\
\hline$B A^{5}$ & $10^{4}$ & 12 & 0.5 & 2.5 & 3 \\
\hline$B A^{10}$ & $10^{8}$ & 18 & 5 & 18 & 23 \\
\hline$B A^{15}$ & $10^{12}$ & 26 & 47 & 76 & 125 \\
\hline$B A^{20}$ & $10^{16}$ & 29 & 522 & 372 & 894 \\
\hline$B A^{25}$ & $10^{20}$ & 30 & 3722 & 1131 & 4853 \\
\hline$T R^{5}$ & $10^{2}$ & 6 & 0.2 & 0.3 & 0.5 \\
\hline$T R^{10}$ & $10^{5}$ & 7 & 13 & 2 & 15 \\
\hline$T R^{15}$ & $10^{7}$ & 10 & 470 & 10 & 480 \\
\hline$T R^{20}$ & $10^{9}$ & 33 & 2743 & 173 & 2916 \\
\hline$T R^{25}$ & $10^{11}$ & 53 & 22107 & 2275 & 24382 \\
\hline
\end{tabular}

Fig. 6. Experimental results of the symbolic heuristic.

reachable in the presence of faults). First, we add all possible transitions that start from $T-S$ and end in $T$. Then, we apply the Algorithm Add_LeadsTo for property $(T-S)$ leads-to $S$.

Figure 6 illustrates experimental results of our heuristic for adding such recovery. All experiments are run on a PC with a $2.8 \mathrm{GHz}$ Intel Xeon processor and 1.2GB RAM. The BDD representation of the Boolean formulae has been done using the Glu/CUDD package ${ }^{2}$. Our experiments target addition of recovery to two well-known problems in fault-tolerant distributed computing, namely, the Byzantine agreement problem [14] (denote $B A^{i}$ ) and the token ring problem [2] (denoted $T R^{i}$ ), where $i$ is the number of processes. Figure 6 shows the size of reachable states in the presence of faults, memory usage, total time spent to add the desirable leads-to property, time spent for cycle detection (i.e., Line 4 in Figure 5), and time spent for breaking cycles by pruning transitions. Given the huge size of reachable states and complexity of structure of programs in our experiments, we find the experimental results quite encouraging. We note that the reason that $T R$ and $B A$ behave differently as their number of processes grow is due to their different structures, existing cycles, and number of reachable states. In particular, the state space of $T R$ is highly reachable and its original program has a cycle that includes all of its legitimate states. This is not the case in $B A$. We also note that in case of $T R$, the symbolic heuristic presented in this subsection tend to be slower than the constructive layered approach introduced in [4]. However, the approach in this paper is more general and has a better potential of success than the approach in [4].

\footnotetext{
${ }^{2}$ Colorado University Decision Diagram Package, available at http://vlsi. colorado.edu/ fabio/CUDD/cuddIntro.html.
} 


\section{Related Work}

The most relevant work to this paper proposes automated transformation techniques for adding UNITY properties to centralized programs [8]. The authors show that addition of multiple UNITY safety properties along with a single progress property to a centralized program can be accomplished is polynomialtime. They also show that the problem of simultaneous addition of two leads-to properties to a centralized program is NP-complete. Also in this context, Jobstmann et al. [11] independently show that the problem of repairing a centralized program with respect to two progress properties in NP-complete.

Existing synthesis methods in the literature mostly focus on deriving the synchronization skeleton of a program from its specification (expressed in terms of temporal logic expressions or finite-state automata) $[1,3,9,15,16]$. Although such synthesis methods may have differences with respect to the input specification language and the program model that they synthesize, the general approach is based on the satisfiability proof of the specification. This makes it difficult to provide reuse in the synthesis of programs, i.e., any changes in the specification require the synthesis to be restarted from scratch.

Algorithms for automatic addition of fault-tolerance to distributed programs are studied from different perspectives $[4,12,13]$. These (enumerative and symbolic) algorithms add fault-tolerance concerns to existing programs in the presence of faults, and guarantee not to add new behaviors to the input program in the absence of faults. Most problems in addition of fault-tolerance to distributed programs are known to be NP-complete.

\section{Conclusion and Future Work}

In this paper, we concentrated on automated techniques for revising finite state distributed programs with respect to UNITY properties. We showed that unlike centralized programs, the revision problem for distributed programs with respect to only one safety or one progress property is NP-complete. Thus, the results in this paper is a theoretical evidence to the belief that designing distributed programs is strictly harder than centralized programs even in the context of finite state systems. Our NP-completeness results also generalize the results in $[12,13]$ in the sense that the revision problems remain NP-complete even if the input program is not subject to faults. We also introduced and implemented a BDDbased heuristic for adding a leads-to property to distributed programs in our tool SYCRAFT [5]. Our experiments show encouraging results paving the path for applying automated techniques for deriving programs that are correct-byconstruction in practice.

For future work, we plan to generalize the issue of distribution by incorporating communication channels in addition to read/write restriction. We also plan to identify sub-problems where one can devise sound and complete algorithms that add UNITY properties to distributed programs in polynomial-time. We also plan to devise heuristics for adding other types of UNITY properties 
to distributed programs. Another interesting direction is to study the revision problem where programs are allowed to have a combination of fair and unfair computations. We conjecture that this generalization makes the revision problem more complex.

\section{References}

1. A. Arora, P. C. Attie, and E. A. Emerson. Synthesis of fault-tolerant concurrent programs. In Principles of Distributed Computing (PODC), pages 173-182, 1998.

2. A. Arora and S. S. Kulkarni. Component based design of multitolerant systems. IEEE Transactions on Software Engineering, 24(1):63-78, 1998.

3. P. Attie and E. A. Emerson. Synthesis of concurrent programs for an atomic read/write model of computation. ACM Transactions on Programming Languages and Systems (TOPLAS), 23(2):187 - 242, 2001.

4. B. Bonakdarpour and S. S. Kulkarni. Exploiting symbolic techniques in automated synthesis of distributed programs with large state space. In IEEE International Conference on Distributed Computing Systems (ICDCS), pages 3-10, 2007.

5. B. Bonakdarpour and S. S. Kulkarni. SYCRAFT: A tool for synthesizing faulttolerant distributed programs. In Concurrency Theory (CONCUR), pages 167-171, 2008.

6. R. E. Bryant. Graph-based algorithms for Boolean function manipulation. IEEE Transactions on Computers, 35(8):677-691, 1986.

7. K. M. Chandy and J. Misra. Parallel program design: a foundation. AddisonWesley Longman Publishing Co., Inc., Boston, MA, USA, 1988.

8. A. Ebnenasir, S. S. Kulkarni, and B. Bonakdarpour. Revising UNITY programs: Possibilities and limitations. In On Principles of Distributed Systems (OPODIS), pages 275-290, 2005.

9. E. A. Emerson and E. M. Clarke. Using branching time temporal logic to synthesize synchronization skeletons. Science of Computer Programming, 2(3):241-266, 1982.

10. E. A. Emerson and C. L. Lei. Efficient model checking in fragments of the propositional model mu-calculus. In Logic in Computer Science (LICS), pages 267-278, 1986.

11. B. Jobstmann, A. Griesmayer, and R. Bloem. Program repair as a game. In Computer Aided Verification (CAV), pages 226-238, 2005.

12. S. S. Kulkarni and A. Arora. Automating the addition of fault-tolerance. In Formal Techniques in Real-Time and Fault-Tolerant Systems (FTRTFT), pages 82-93, 2000.

13. S. S. Kulkarni and A. Ebnenasir. The complexity of adding failsafe fault-tolerance. International Conference on Distributed Computing Systems (ICDCS), pages 337344, 2002.

14. L. Lamport, R. Shostak, and M. Pease. The Byzantine generals problem. ACM Transactions on Programming Languages and Systems (TOPLAS), 4(3):382-401, 1982.

15. Z. Manna and P. Wolper. Synthesis of communicating processes from temporal logic specifications. ACM Transactions on Programming Languages and Systems (TOPLAS), 6(1):68-93, 1984.

16. A. Pnueli and R. Rosner. On the synthesis of a reactive module. In Principles of Programming Languages (POPL), pages 179-190, 1989. 